\title{
Depresión y adherencia a la medicación antirretroviral: efectos de una intervención conductual breve ${ }^{1}$
}

\section{Depression and adherence to antiretroviral medication: Effects of a brief behavioral intervention}

\author{
Humberto Valle-Soto², Susana Robles-Montijo ${ }^{3}$, \\ John S. Wiebe y Juan José Sánchez-Sosa ${ }^{2}$
}

Citación: Valle S., H., Robles M., S., Wiebe, J.S. y Sánchez-Sosa, J.J. (2019). Depresión y adherencia a la medicación antirretroviral: efectos de una intervención conductual breve. Psicología y Salud, 29(1), 139-153.

\section{RESUMEN}

\begin{abstract}
Antecedentes. Hay una considerable ausencia de intervenciones sencillas y eficaces para aumentar los niveles de adherencia a la terapia antirretroviral y disminuir los niveles de depresión en población seropositiva mexicana. Objetivo. En este contexto, se evaluaron los efectos de una intervención basada en las técnicas de activación conductual, adicionada con módulos de psicoeducación, solución de problemas y relajación muscular progresiva profunda, sobre los niveles de sintomatología depresiva y adherencia a la terapia antirretroviral de pacientes seropositivos de la Ciudad de México. Método. Se usó un diseño intrasujetos con ocho réplicas individuales y mediciones de línea base, postratamiento y seguimiento a un mes sobre variables psicológicas y conductas de adhesión medidas mediante el VPAD-24, el BDI-II, el SVE-12 y los autorregistros conductuales de la toma de medicamento y el nivel de agrado de las actividades. Resultados. Los resultados se presentan en tablas de resumen y gráficas para cada participante según la etapa de intervención. Discusión. La combinación de procedimientos disminuyó los niveles de sintomatología depresiva y aumentó los niveles de adherencia. La activación conductual y la solución de problemas se aprecian más asociadas a un aumento del nivel de agrado y de toma del medicamento antirretroviral en los autorregistros. En consecuencia, es posible proponer la conclusión preliminar de que la intervención propuesta disminuye los niveles de depresión, aumenta los niveles de adherencia a la terapia antirretroviral y mejora el manejo de situaciones generadoras de estrés.
\end{abstract}

Palabras clave: VIH; Depresión; Estrés; Adherencia terapéutica; Terapia conductual.

\begin{abstract}
Background. There is a noticeable scarcity of publications on easy-to-administer interventions aimed at increasing therapeutic adherence and decreasing depressive symptoms in persons living with HIV in Latin America in general, and in Mexico in particular. Objective. To evaluate the clinical effects on depressive symptoms and medication adherence, of an intervention based on behavioral activation techniques, supplemented with educational materials, problem-solving strategies and deep muscle relaxation. Method. A within-subjects baseline-pre-post-follow-up design with eight individual clinical replications was used to examine the effects of the treatment. Measures included Beck's Depression Inventory, the VPAD-24 and the SVE-12 scales for adherence and stress management respectively, and additional behavioral self-recording techniques on medication adherence
\end{abstract}

\footnotetext{
${ }^{1}$ Los autores agradecen muy especialmente a los doctores Laura Acuña y Víctor Rodríguez sus comentarios a una versión preliminar del manuscrito. El presente estudio recibió apoyo financiero parcial de las subvenciones 248477 del Consejo Nacional de Ciencia y Tecnología, e IN-303618 de la Dirección de Asuntos del Personal Académico e Investigación de la UNAM (PAPIIT). Artículo recibido el 14 de agosto y aceptado el 3 de septiembre de 2018.

${ }^{2}$ Facultad de Psicología, Universidad Nacional Autónoma de México, Av. Universidad 3004, Edif. "D” Mezzanine, Laboratorio 015, Col. Copilco Universidad, Del. Coyoacán, 04510 Ciudad de México, México, correos electrónicos: hvalleso@gmail.com. y jujosaso@gmail.com.

${ }^{3}$ Facultad de Estudios Superiores Iztacala, Universidad Nacional Autónoma de México, Av. de Los Barrios 1, Los Reyes Iztacala, 52970 Tlalnepantla, Edo. de México, México, correo electrónico: susana@unam.mx.

${ }^{4}$ Psychology Building, Room 112, Department of Psychology, The University of Texas at El Paso, 500 W. University, 79902 El Paso, TX, Estados Unidos de América, correo electrónico: jwiebe@utep.edu.
} 
and perceived pleasing value of behavioral activation behaviors and activities. Treatment integrity was protected through standardized materials and consistent therapeutic techniques. Results. Data analyses show clinically relevant decreases in depressive symptoms as well as increases in medication adherence and perceived pleasing value of behavioral activation actions in all participants, as shown by scales' data and self-behavioral recordings. Discussion. Although some methodological precautions could well improve future similar studies, the present findings suggest that an easy-to-administer combination of behavioral activation and didactics-based exercises and planning are effective interventions on HIV+ Spanish-speaking participants.

Key words: HIV; Medication; Depression; Behavioral activation; Therapy.

$\mathrm{E}$ 1 virus de inmunodeficiencia humana (VIH) es un retrovirus que ataca el sistema inmunológico de las personas (Castro, Gutiérrez y Villena, 2007) y ocasiona un deterioro progresivo del sistema inmunológico (Klimas, O'Brien y Fletcher, 2008; Organización Mundial de la Salud [OMS], 2013); Vargas y Boza, 2012).

El VIH sigue siendo uno de los principales problemas de salud pública en el mundo; en el año 2016 había aproximadamente 36.7 millones de personas infectadas, y ese mismo año se produjeron 1.8 millones de nuevas infecciones y un millón de personas fallecieron por causas relacionadas con el virus (OMS, 2017). En México, el número de casos de VIH muestra un aumento importante año tras año; a la fecha, hay unos 73,215 pacientes con VIH, de los cuales 13,247 residen en la Ciudad de México (Centro Nacional para la Prevención y el Control del VIH y el Sida [CENSIDA], 2017; Secretaría de Salud/CENSIDA, 2008).

Actualmente, la infección por VIH podría concebirse como una infección crónica con una esperanza de vida casi normal (Castro et al., 2007; World Health Organization [WHO], 2016) gracias a la terapia con medicamentos antirretrovirales, cuyo objetivo es disminuir el número de copias del virus a una medida "indetectable" -esto es, por debajo de 50 copias por mililitro de sangre (Hammer et al., 2006)- y aumentar los conteos de células protectoras CD4 en la sangre del paciente (Castro et al., 2007; U.S. Department of Health and Human Services, 2017; Vargas y Boza, 2012).
Se ha demostrado que el éxito de la terapia antirretroviral depende de que el paciente observe niveles tan elevados como $95 \%$ de apego a las pautas de la medicación para mantener la replicación viral en un nivel mínimo. Una disminución minúscula de la adherencia (tan pequeña como de 95 a 90\%) suele resultar en una reducción marcada de las tasas de supresión viral (Berg, Michelson y Safren, 2007; Castro et al., 2007; Shuter, 2008; wHO, 2016). Pese a su importancia, en México se ha encontrado que solo alrededor de la mitad de los pacientes muestra una adherencia óptima (arriba de 95\%) (Balandrán, Gutiérrez y Romero, 2013; Peñarrieta et al., 2009; Piña, Dávila, Sánchez-Sosa, Togawa y Cázares, 2008).

Diversos estudios han mostrado la correlación inversa que existe entre la depresión y la adherencia terapéutica (DiMatteo, Lepper y Croghan, 2000; Horberg et al., 2008; Maru et al., 2008; Orejudo, Ladero, Carrobles y Malo, 2006; Piña et al., 2008). Por ejemplo, Uthman, Magidson, Safren y Nachega (2014) realizaron un metaanálisis que incluía 111 estudios sobre la relación entre ambas variables en una muestra total de 42,366 participantes. Concluyeron que la probabilidad de sostener una adherencia adecuada (toma $\geq 95 \%$ ) era $42 \%$ menor en los participantes que mostraban síntomas depresivos que en aquellos que no los padecían.

Aproximadamente un tercio de los pacientes manifiestan sintomatología depresiva (entre 20 y 40\% de los casos) (Galvan, Davis, Banks y Bing, 2008; Horberg et al., 2008; Mao et al., 2008; Newman, Limin, Kippax, Saltman y Kidd, 2008; Pinto et al., 2012). En México se ha visto una prevalencia muy semejante a la encontrada en estudios internacionales; por ejemplo, Soto y Cruz (2014), trabajando con una muestra de 252 participantes de Ciudad Juárez, hallaron que, medida con el Inventario de Depresión de Beck (BDI), 20.6\% de ellos padecían depresión. Galindo y Ortega (2010), en Xalapa, encontraron que $18.76 \%$ de un total de 160 pacientes padecían depresión, medida con el Cuestionario Clínico de Síndrome Depresivo (Calderón, 1995). Cruz (2015), en Ciudad de México, halló en una muestra de 223 participantes que $26 \%$ de ellos padecían síntomas depresivos medidos con el BDI.

En dichos pacientes se observa una estrecha relación entre la adherencia terapéutica, la depresión 
y el estrés. La aparición de estados emocionales tales como la ansiedad o el estrés está relacionada con la probabilidad de padecer sintomatología depresiva (Rodkjaer, Laursen, Balle y Sodemann, 2010). En uno de esos estudios, Bottonari, Roberts, Ciesla y Hewitt (2005) hallaron que ciertos eventos estresantes pueden ocasionar lapsos de escasa adherencia, aun en ausencia de sintomatología depresiva; sin embargo, dicho efecto es mucho mayor cuando el paciente se encuentra deprimido. Por su parte, el modelo propuesto por Piña y Sánchez-Sosa (2007) y Sánchez-Sosa, Cázares, Piña y Dávila (2009) concede una posición relevante al estrés como un factor que afecta la adherencia terapéutica.

La terapia cognitivo-conductual para la adherencia terapéutica y la depresión (CBT-AD, por sus siglas en inglés) ha mostrado ser efectiva para disminuir los niveles de depresión y aumentar los de adherencia en distintos estudios. La CBT-AD se basa en el abordaje tradicional cognitivo-conductual para la depresión y combina diversas técnicas de intervención empleadas en enfermedades crónicas (Safren, Otto y Worth, 1999; Thomason, Bachanas y Campos, 1996). El programa ha demostrado en repetidas ocasiones su efectividad en pacientes (predominantemente blancos no hispanos) con VIH/sida. Numerosos ensayos controlados aleatorizados demuestran los claros efectos de la CBT-AD (Safren et al., 2001; Safren et al., 2009).

Existen pocos intentos por emplear la CBTAD en población latina. Solamente Simoni et al. (2013) han hecho una prueba clínica aleatorizada preliminar de la CBT-AD en 40 participantes de ascendencia latina en la ciudad de El Paso; en dicha prueba, el tamaño del efecto de la intervención fue de moderado a grande, incluso en las mediciones de seguimiento, viéndose aumentada la adherencia, disminuidos los niveles de depresión y mejorados los resultados en mediciones clínicas del grupo intervenido ante uno de comparación.

La activación conductual (AC) es un componente de la CBT-AD que busca mejorar el estado de ánimo de los pacientes mediante el aumento de actividades placenteras, lo que facilita la aparición de reforzadores potencialmente antidepresivos en el ambiente. En general, la AC busca identificar y promover que el paciente realice actividades que le resulten placenteras (Santiago-Rivera et al.,
2008), consistentes con sus metas a largo plazo, con el propósito de romper el ciclo de la depresión (Dimidjian et al., 2006).

La AC por si sola ha mostrado su efectividad para disminuir los niveles de depresión en participantes con otros problemas, y tiene niveles de eficacia tan elevados en pacientes con depresión grave como los obtenidos con antidepresivos, y aún mejores que los de la terapia cognitiva sola (Dimidjian et al., 2006). La AC genera una considerable resistencia a las recaídas, equiparable a los de las terapias cognitiva y cognitivo-conductual (Gortner, Gollan, Dobson y Jacobson, 1998), e incluso más elevada que el tratamiento con antidepresivos (Dimidjian et al., 2006). En distintas revisiones y metaanálisis no se encuentran diferencias significativas entre la terapia cognitivo-conductual con o sin las técnicas cognitivas (Bell, Marcus y Goodlad, 2013).

Específicamente en poblaciones latinas, las intervenciones con AC han obtenido resultados positivos para disminuir la sintomatología depresiva (Kanter, Diéguez, Rusch, Busch y Santiago-Rivera, 2008), por lo que se prefiere su uso al de las intervenciones cognitivo-conductuales tradicionales (Santiago-Rivera et al., 2008). Los componentes de la AC se adaptan fácilmente a los valores culturales de las poblaciones latinas y tienen un potencial mayor que las intervenciones cognitivo-conductuales (Santiago-Rivera et al., 2008). Un ensayo clínico realizado por Simoni et al. (2013) mostró que los pacientes calificaron los componentes de la $\mathrm{AC}$ como los más aceptados y útiles.

Respecto al VIH, Magidson, Seitz-Brown, Safren y Daughters (2014) realizaron una intervención grupal cuyo contenido era flexible, pues sólo se contaba con los módulos de AC y el ejercicio de planeación denominado pasos vitales. Participaron 13 pacientes, 12 de los cuales eran afroamericanos, a quienes se dio un promedio de 3.49 sesiones por paciente (el programa constaba de ocho sesiones). Al concluir la intervención, se observó una reducción significativa de la depresión, medida con la Escala de Ansiedad y Depresión Hospitalaria (HADS). Sin embargo, dichas mediciones solo se hicieron en cuatro participantes, los únicos que decidieron acudir de forma voluntaria a las sesiones de seguimiento. Dos de ellos fueron los que 
más habían asistido a las sesiones grupales. Además, se vio una ligera mejoría en los niveles de adherencia de los pacientes, no obstante lo cual ya mostraban adherencia desde la línea base.

Tomando en cuenta ambas intervenciones (CBT-AD y AC), se propone que sí los componentes cognitivos relativamente complejos de la CBTAD pueden omitirse sin una pérdida significativa de la eficacia en el tratamiento, la intervención conductual derivada podría ser ampliamente aceptada por los pacientes latinos y muy fácilmente aplicable a través de una intervención hecha por el personal sanitario no especializado. También tendría el potencial de ser extendida a consejeros paraprofesionales, como trabajadores sociales y otros empleados del sector salud.

Hay, en efecto, pocos estudios que evalúen la eficacia de las intervenciones para mejorar la adherencia y disminuir la sintomatología depresiva y el estrés en pacientes seropositivos en México. Por tanto, el objetivo del presente estudio fue evaluar los efectos de la terapia de AC en los niveles de carga viral plasmática (CV), conteos de CD4, adherencia al tratamiento antirretroviral, sintomatología depresiva y afrontamiento ante el estrés en pacientes con VIH/sida de la Ciudad de México.

\section{MÉTODO}

\section{Diseño}

En virtud de tratarse de un diseño experimental ampliamente documentado en la literatura de investigación en psicología y considerado como especialmente sólido en estudios clínicos, el presente estudio siguió un diseño intrasujetos, con tantas réplicas clínicas como participantes se logró reunir para el estudio, de manera que cada participante representa una réplica sistemática del mismo (Kazdin, 2011; Sidman, 1960; Tate et al., 2013).

\section{Participantes}

El estudio incluyó participantes varones mayores de 18 años de edad de la referida ciudad con VIH/ sida que se encontraban bajo un tratamiento antirretroviral en ese momento. Los participantes exhibían una sintomatología depresiva indicada por puntajes superiores a 13 en el IDB-II, y una adherencia subóptima a la terapia antirretroviral (menor a 95\% en la última semana), tal como proponen Golin et al. (2002) con el reactivo "En los últimos siete días, ¿cuántas veces no ha tomado su medicamento?". Se tomó como error de adherencia cualquier desviación en el horario de ingestión del medicamento antirretroviral o la omisión de las tomas reportadas por el paciente.

No participaron en el estudio pacientes con evidencia o diagnóstico formal de psicosis activa, uso de drogas inyectables, cáncer o deterioro cognitivo importante en virtud de que son condiciones que imposibilitan la instrumentación de los procedimientos educativo-clínicos. En ausencia de un diagnóstico formal, se empleó el cribado y juicio del clínico tratante. Se excluyó del análisis final a quienes no completaron $80 \%$ de los contenidos de la intervención, particularmente el módulo de AC.

Se reclutó a los participantes mediante invitaciones en línea, tanto en redes sociales como en grupos de apoyo para pacientes con VIH/sida, y se pidió a esos participantes activos que invitaran a otros posibles interesados en sumarse al estudio. Por último, se acudió a distintas organizaciones y centros de apoyo no gubernamentales (Fundación Unidos por un México Vivo, A.C. y Fundación Eudes) para repartir trípticos y carteles en distintos puntos de la ciudad en los que se invitaba a participar. Una vez contactados los mismos, se les hacía una entrevista inicial para valorar el cumplimiento de los criterios de inclusión.

Hubo doce participantes que satisficieron las características anteriormente descritas, con una media de edad de 27.5 (22-35 años), de los cuales ocho completaron el estudio. Tres de ellos no poseían un trabajo formal, uno era estudiante, seis estaban en una relación de pareja estable, tres de los cuales vivían con sus parejas, todos habían concluido el bachillerato, uno estudiaba una licenciatura, otro tenía estudios de maestría y el resto (seis) tenían el grado de licenciatura. 


\section{Validación por expertos y pilotaje}

El manual de tratamiento fue evaluado de manera independiente por cinco especialistas en VIH: dos psiquiatras y tres psicólogos con posgrado, y las técnicas de intervención cognitivo-conductuales por dos psicólogos con doctorado y un especialista en desarrollo de manuales educativos. Los contenidos gráficos y autorregistros empleados fueron revisados en jueceo independiente por 22 psicólogos especialistas en ciencias conductuales y de la salud, a fin de corroborar su pertinencia para la recolección de datos y su uso a lo largo de la intervención.

Hecho lo anterior, se llevó a cabo un pilotaje del tratamiento con dos participantes, tras de lo cual el equipo de investigación hizo los cambios necesarios a los materiales y técnicas de la intervención con vistas a su integridad, idoneidad y comprensión para su uso en el estudio.

\section{Entrenamiento y supervisión de terapeutas}

La intervención fue administrada por un psicólogo con grado de maestría con base en los materiales desarrollados en el proyecto de la Universidad de Texas en El Paso (Simoni et al., 2013), adaptados al castellano de la Ciudad de México, mediante los procedimientos descritos arriba. Además del entrenamiento específico para guiarse por el protocolo de intervención y los procedimientos prescritos por los materiales, cada paciente evaluó los contenidos de cada uno de los módulos al final de cada sesión a fin de valorar la integridad del tratamiento.

\section{Medición}

Se tomaron los datos sociodemográficos de los participantes (edad, estado civil, ocupación, nivel educativo y situación laboral), el tiempo transcurrido desde el diagnóstico de VIH y la pauta médica prescrita. Se recolectaron los resultados de las pruebas de laboratorio, de los cuales se capturaron la cantidad de linfocitos CD4 en sangre (conteos de CD4) y la cantidad de copias de RNA del VIH en sangre expresada en copias/ml de sangre (CV).

Para el resto de variables se emplearon instrumentos psicométricos o autorregistros conductuales del modo en que se describe a continuación.
Adherencia terapéutica a la terapia antirretroviral: Puntajes obtenidos en el cuestionario de adherencia terapéutica a antirretrovirales: variables psicológicas y comportamientos de adhesión (VPAD-24) y mediciones realizadas mediante autorregistros. El VPAD-24 posee validez conceptual, concurrente y predictiva adecuadas (Piña, Corrales, Mungaray y Valencia, 2006), y contiene 24 preguntas tipo Likert agrupadas en cuatro apartados y con alta consistencia interna $(\alpha=0.852)$.

Sintomatología depresiva: Puntajes obtenidos mediante el IDB-II adaptado a población mexicana. La escala muestra consistencia interna $(\alpha=.87$ a .92) y cumple con los criterios de validez convergente con las escalas de depresión y ansiedad del HADS. La versión mexicana, al igual que la original, se compone por tres factores de 21 reactivos con cuatro opciones de respuesta tipo Likert. Los puntajes resultantes categorizan el nivel de depresión como mínimo, leve, moderado y grave (González, Reséndiz y Reyes, 2015).

Activación conductual: Media semanal del nivel de agrado diario referido en las mediciones de autorregistros o autoinforme de actividades tipificadas por el propio participante como agradables. El autorregistro incluye el día de la semana, las actividades realizadas a lo largo del día, el nivel de agrado general por las actividades llevadas a cabo y el nivel de agrado del día.

Afrontamiento del estrés: Puntajes obtenidos en la Escala de Situaciones Vinculadas con el Estrés (SVE-12). Esta escala, adaptada a población seropositiva (Piña, Valencia, Mungaray y Corrales, 2006), posee validez de constructo y concurrente y una alta consistencia interna ( $\alpha=0.904)$. Consta de doce preguntas tipo Likert, agrupadas en tres factores. Las respuestas se muestran en formato Likert y van de 1 ("No es una situación amenazante") a 10 ("Es una situación sumamente amenazante").

Se efectuaron mediciones de las variables a lo largo de todo el estudio; los autorregistros se emplearon todas las semanas a partir de la línea base, misma que duró de dos a tres, hasta la semana posterior a la última sesión, y las semanas necesarias para medir el seguimiento. Los instrumentos psicométricos se utilizaron en cuatro momentos distintos: al inicio de línea base, al empezar el tratamiento, al concluir éste y al mes de seguimiento. 


\section{Procedimiento}

Luego de obtenerse su línea base, los participantes empezaban la etapa de intervención, la cual comprendió siete módulos de terapia basada en técnicas de AC de manera individual con el terapeuta. Dichos módulos incluyen los utilizados por Berg, Raminani, Greer, Harwood y Safren (2008), Safren et al. (2009), Safren et al. (2012) y Simoni et al. (2013). Se agregó un módulo de retención en el que el paciente enunciaba sus principales razones para continuar con el tratamiento y no suspenderlo.

Los demás componentes incluyeron psicoeducación sobre sintomatología depresiva, activación conductual basada en la empleada por Daughters et al. (2008), Gawrysiak, Nicholas y Hopko (2009) y Daughters, Magidson, Schuster y Safren (2010); entrenamiento de adherencia (Pasos Vitales), basado en Safren et al. (2009), Safren et al. (2012) y Simoni et al. (2013), con un formato simple de solución de problemas, y entrenamiento en respiración diafragmática y relajación muscular con el protocolo de Fahrion y Norris (1990) y Garduño, Riveros y Sánchez-Sosa (2010).

\section{Repaso y prevención de recaídas}

Aunado a las sesiones presenciales realizadas cada dos semanas, se hicieron llamadas telefónicas de seguimiento a los participantes en las semanas de descanso. En esas llamadas se revisaba brevemente el módulo de retención -particularmente si habían incluido alguna razón más para continuar en el tratamiento-; se evaluaba la adherencia de la semana anterior mediante la pregunta "En los últimos siete días, ¿cuántas veces no ha tomado su medicamento?"; se daba retroalimentación sobre la adherencia, tanto si habían observado una adherencia adecuada (reforzamiento social positivo), como si habían cometido errores en ella (“¿Por qué motivo no logramos la toma completa del medicamento?"), y por último se evaluaban su posibles dudas al elaborar los registros y llevar a cabo las tareas en casa.

Las sesiones tenían una duración de una a dos horas cada catorce días, o con la frecuencia permitida por la disponibilidad temporal de los participantes, pues se buscaba completar el tratamiento en aproximadamente dos meses. Se permitió la flexibilidad en el número de sesiones dedicadas a cada uno de los módulos para que los participantes se adaptaran a la complejidad clínica de cada caso (Safren et al., 2009; Safren et al., 2012; Simoni et al., 2013). Sin embargo, el objetivo era que la intervención se concluyera, de ser posible, en solamente cuatro sesiones.

\section{RESULTADOS}

La Figura 1 muestra los puntajes de adherencia al régimen antirretroviral de cada participante.

Figura 1. Porcentaje de adherencia promedio por participante por periodo del estudio.

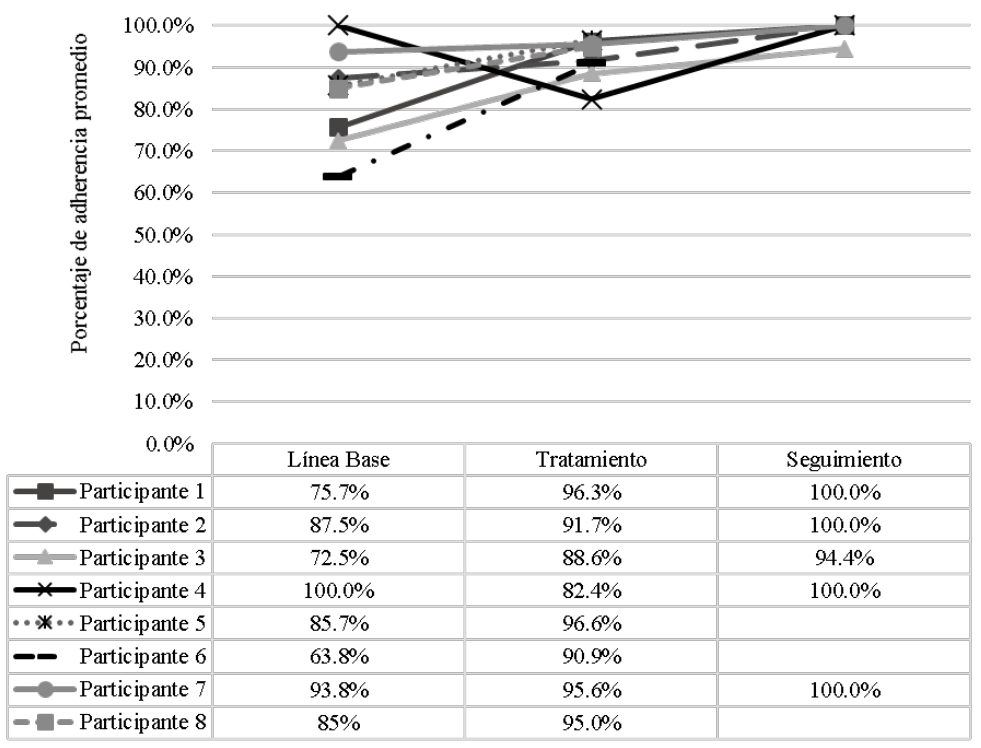


Dichos porcentajes incluyen las fallas en la toma del medicamento en horarios adecuados, en un esquema de representación típica (el paciente más beneficiado, el menos beneficiado y al que más se parecían los datos del resto). Los puntajes del participante 6 muestran los menores niveles iniciales de adherencia: de 63.8 a $90.9 \%$ al concluir (27.1\% de mejoría, aunque claramente aún insuficiente). El participante 3 representa al paciente típico, es decir, la forma en que se comportó la mayoría de los pacientes; inicia el periodo de línea base con una adherencia de $72.5 \%$, aumenta a $88.6 \%$ durante el periodo de intervención y concluye el seguimiento con $94.4 \%$ (21.5\% de mejo- ría, prácticamente en la meta clínica). Por último, el participante 1 fue el que más aumentó sus niveles de adherencia, pasando de 75.7 a 100\% (adherencia perfecta) en las mediciones de seguimiento (24.3\% de mejoría).

La Tabla 1 muestra cómo los participantes 4, 5 y 8 redujeron a niveles indetectables su carga viral a lo largo del estudio. Dichos participantes, además, eran de reciente diagnóstico, a diferencia del participante 6, el cual reportó en la primera medición una carga viral de 67 copias por mililitro de sangre, para después pasar a niveles indetectables.

Tabla 1. Pauta médica prescrita y resultados de pruebas de sangre por participante a lo largo del estudio.

Participantes

\begin{tabular}{|c|c|c|c|c|c|c|c|c|}
\hline & 1 & 2 & 3 & 4 & 5 & 6 & 7 & 8 \\
\hline Edad & 25 años & 23 años & 37 años & 24 años & 22 años & 29 años & 25 años & 35 años \\
\hline $\begin{array}{l}\text { Medicación } \\
\text { prescrita }\end{array}$ & Atripla & Atripla & Atripla & $\begin{array}{c}\text { Truvada } \\
\text { Dolutegravir }\end{array}$ & $\begin{array}{c}\text { Truvada } \\
\text { Dolutegravir }\end{array}$ & Atripla & Atripla & $\begin{array}{c}\text { Truvada } \\
\text { Dolutegravir }\end{array}$ \\
\hline $\begin{array}{l}\text { Tiempo de } \\
\text { diagnóstico }\end{array}$ & $\begin{array}{c}1 \text { año } \\
\text { y medio }\end{array}$ & 2 años & 7 años & 9 meses & 2 meses & 5 años & 4 meses & 6 meses \\
\hline $\mathrm{CV}-\mathrm{LB}$ & Indetectable & Indetectable & Indetectable & 1200 & $29 ’ 100$ & 67 & $1 ’ 514$ & 84 \\
\hline CD4 -LB & 957 & 768 & 1107 & 435 & 366 & 657 & 485 & 203 \\
\hline CV - Intv. & & & & & & & 114 & Indetectable \\
\hline CD4 - Intv. & & & & & & & 735 & 554 \\
\hline $\begin{array}{l}\mathrm{CV}- \\
\text { Seguimiento }\end{array}$ & Indetectable & Indetectable & Indetectable & Indetectable & Indetectable & Indetectable & Indetectable & \\
\hline $\begin{array}{l}\text { CD4 - } \\
\text { Seguimiento }\end{array}$ & 1108 & 913 & 870 & 503 & 985 & 715 & - & \\
\hline
\end{tabular}

Nuevamente, en términos de representatividad típica, la Figura 2 muestra los datos del promedio semanal de agrado con las actividades entre los participantes 4,7 y 8 , según sus autorregistros de activación conductual. El participante 8 fue el que más se benefició de la intervención, el participan- te 7 el que menos, y el participante 4 representa cómo se comportó la mayoría de las puntuaciones en el resto de participantes.

A su vez, la Tabla 2 detalla los puntajes de todos los participantes. 
Figura 2. Promedio de nivel de agrado semanal por semana y por periodo durante el estudio.

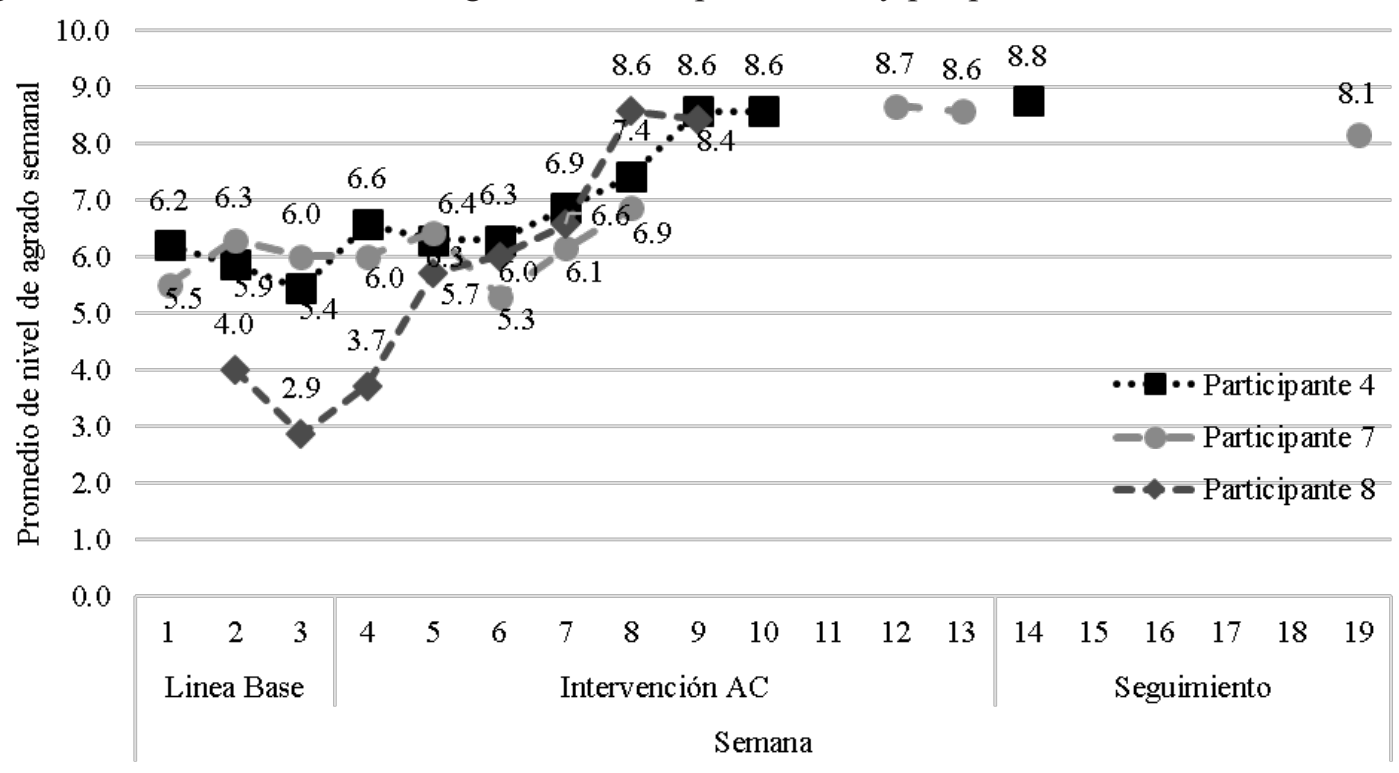

Tabla 2. Promedios de nivel de agrado semanal por participante por periodo.

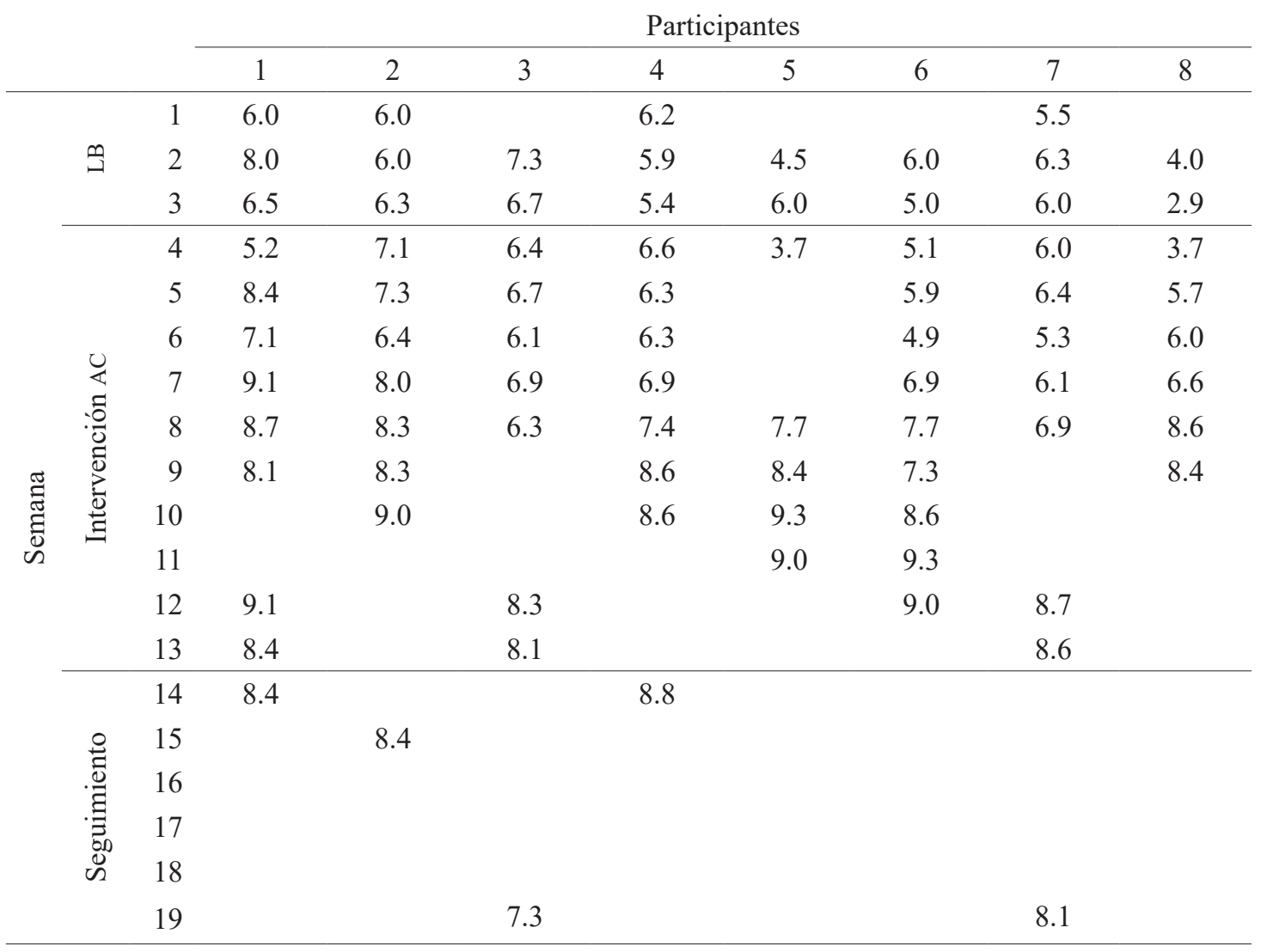

La tabla anterior muestra que los mayores cambios ocurrieron en los participantes 2, 4, 5 y 8, los cuales presentan las diferencias más grandes entre el promedio de sus puntajes de línea base y sus mediciones de seguimiento; para el participante 8, es entre el promedio de línea base y el promedio durante el periodo de intervención; con cambios menores están los participantes 1 y 6 , y la menor mejoría fue la del participante 3 , que pasa de un promedio de 7 en la línea base a una de 7.3 en el seguimiento.

La Figura 3 muestra cómo todos los participantes iniciaron la línea base con depresión leve o superior, según los puntajes del instrumento. 
Figura 3. Sintomatología depresiva por etapa de investigación.

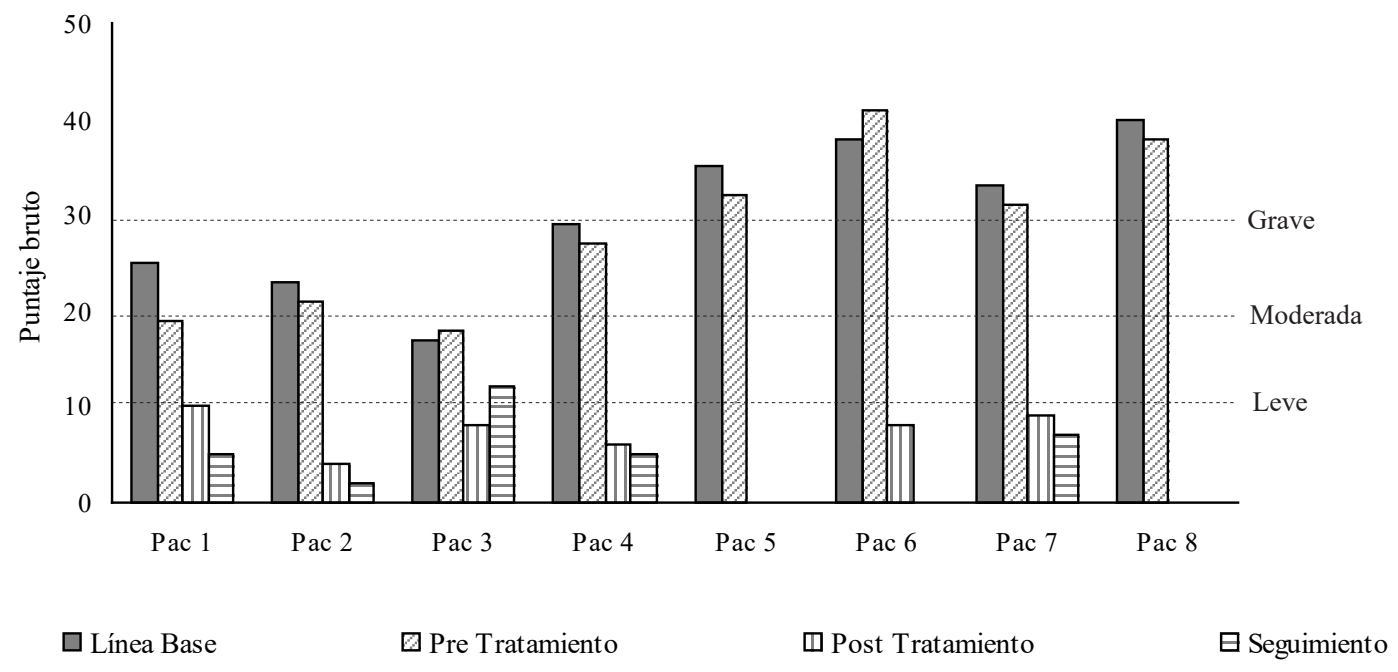

En la figura se aprecia que el participante 1 comienza el tratamiento por debajo de su puntuación de línea base, pasando de depresión moderada a leve, tendencia que se mantiene hasta el seguimiento. Sólo el participante 3 no alcanza puntaciones por arriba de la depresión leve en la línea base, las que se reducen a diez puntos y aumentan ligeramente en el seguimiento. Los participantes 5, 6, 7 y 8 puntuaron como depresión grave en línea base, $\mathrm{y}$ únicamente los participantes 6 y 7 muestran niveles postratamiento con puntajes por debajo del punto de corte para depresión leve.

Por último, la Figura 4 muestra las mediciones de todos los participantes. Se observa una mejoría al comparar las puntuaciones en línea base y pretratamiento con las posteriores (a menor puntaje bruto, menor nivel de estrés).

Figura 4. Sintomatología por estrés percibido.

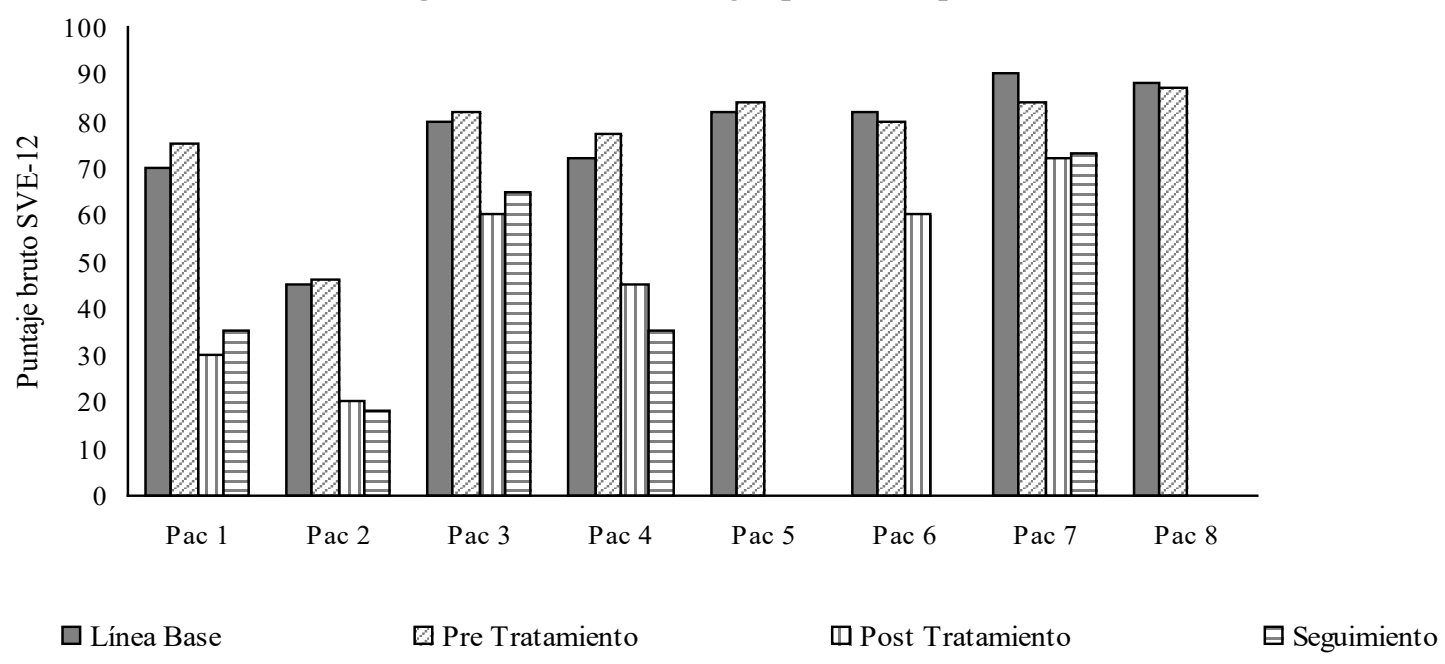

\section{DISCUSIÓN}

Los resultados hallados permiten proponer que, al igual que en otras enfermedades crónicas (Hopko et al., 2013; Lundervold, Talley y Buermann, 2008; Magidson et al., 2014), la activación conductual disminuye la sintomatología depresiva. La intervención también aumenta los niveles de adheren- cia médica de los participantes y disminuye los de estrés percibido.

Si bien este resultado se aprecia en todos los participantes, al igual que en Magidson et al. (2014), estos resultados son limitados debido a la cantidad de participantes y a las diversas características clínicas y situacionales. 
Los niveles de adherencia en la línea base ( sin considerar algunas desviaciones menores de horario) indican que el número de participantes con niveles de adherencia óptimos - por arriba de 95\%- es muy similar al hallado en otros estudios, incluidos los llevados a cabo en México (Balandrán et al., 2013; Berg et al., 2007; Peñarrieta et al., 2009; Piña et al., 2008; Shuter, 2008; WHO, 2016). En efecto, en el presente estudio la mitad de los participantes exhibieron niveles superiores a ese porcentaje en la línea base.

Exceptuando el participante 3, todos mejoraron su sintomatología depresiva, pasando de niveles de depresión moderada y grave a niveles por debajo de leve. En cuanto a los resultados del VPAD-30, al comparar línea base con el postratamiento y el seguimiento, no hubo desviaciones de los puntajes mayores a 22 (puntaje bruto), lo que quizá se deba a que gran parte del cuestionario evalúa la motivación a ser adherente y las conductas de adherencia médica previas al diagnóstico de VIH. Por lo tanto, las diferencias, aunque pequeñas, son clínicamente relevantes porque incluyen directamente el estrés percibido, los síntomas depresivos y la adherencia terapéutica.

Si bien un análisis de la contribución relativa de cada módulo está lejos del propósito del presente estudio, algunos hallazgos clínicos específicos complementan los aquí descritos y resultan interesantes. Por ejemplo, los derivados de los reactivos "En el transcurso de la semana pasada, ¿consumió usted todos los medicamentos que le dio su médico?”, “QQué tan difícil se le hace a usted mantenerse relajado y en calma?" y "¿Qué tan difícil se le hace a usted evitar situaciones que le provoquen tristeza o que lo depriman?". Conviene recordar que el SVE-12 evalúa la respuesta de estrés ante situaciones específicas que tienen que ver con el tratamiento del paciente (Piña et al., 2006), lo que hace suponer que el desarrollo de estrategias para afrontar eventos específicos facilita una reducción en respuestas poco útiles para manejar el estrés. Tal posibilidad sugiere que la intervención facilita el desarrollo de competencias funcionales para afrontar situaciones estresantes relacionadas con el VIH.

Los resultados resultan relevantes en cuanto que indican que, en efecto, la activación conductual es una opción sencilla, viable, breve (Hopko,
Lejuez, Ruggiero y Eifert, 2003) y de bajo costo al no requerir la intervención de personal altamente capacitado (Ekers et al., 2011), situación que prevalece por lo general en clínicas y centros públicos de atención en América Latina.

Es muy probable que la intervención haya producido efectos positivos en la adherencia y que, tal como indican Balfour et al. (2006), Gardner, Burman, Steiner, Anderson y Bangsberg (2009) y Hughes et al. (2011), el éxito o fracaso del tratamiento depende de no requerirlo. Del mismo modo, disminuir los niveles de depresión en los pacientes supone evitarles en el largo plazo meses de costoso e innecesario sufrimiento emocional (Valente, 2003).

Luego de la implementación de la activación conductual, se observó un aumento en el nivel de agrado diario de los participantes. Ello indica asimismo una relación entre la implementación del tratamiento y la reducción de la sintomatología depresiva, relación también encontrada por Magidson et al. (2014) en pacientes seropositivos. Este hallazgo parece apoyar las teorías conductuales de la depresión, en el sentido en que, tal como lo explica Ferster $(1965,1973)$, la disminución de los reforzadores positivos contingentes a la conducta de los participantes propicia la aparición de sintomatología depresiva. Es probable que, gracias a la $\mathrm{AC}$, los participantes comenzaran a percibir reforzadores positivos contingentes a su conducta en su propio ambiente, y a contrarrestar la disminución de la efectividad de los reforzadores debido a la pérdida de placer en estados depresivos (Costello, 1972).

Una posible explicación adicional de la mejoría de la sintomatología depresiva podría residir en el módulo de solución de problemas. La solución inadecuada de problemas se ha asociado con la sintomatología depresiva cuando los individuos encaran situaciones difíciles (Nezu, 1987). Esto podría sugerir que una posible explicación a la disminución de tal sintomatología en el presente estudio se pueda también atribuir a los efectos del módulo de solución de problemas (Nezu, Nezu, Saraydarian, Kalmar y Ronan, 1986). Tal sería el caso del participante 2, que mejoró su sintomatología depresiva, estrés percibido y falta de adherencia sólo hasta que encontró formas de afrontar sus problemas económicos, de horario y de pareja. 
Otra explicación de este fenómeno reside tal vez en una transferencia del efecto clínico al medio social natural de los pacientes y a una habituación propiciada por el paso del tiempo desde que principia la activación conductual. Quizá la mejoría de los participantes 2, 6 y 7 pudo deberse al tiempo transcurrido desde la sesión de AC y psicoeducación sobre la depresión, y no únicamente al módulo de solución de problemas. Fue únicamente hasta después de sus vacaciones que el participante 7 mostró un aumento considerable en el promedio de agrado semanal, lo que puede indicar la necesidad de periodos más prolongados para estabilizar claramente los efectos de las actividades agradables en este participante.

En las gráficas presentadas se aprecia cómo los cambios en algunos de los participantes permiten suponer que, a partir del uso de estrategias adecuadas para mantenerse adherentes (en los módulos de solución de problemas y pasos vitales), lograron niveles de adherencia altos que se mantuvieron por varias semanas. Los participantes 1 , 2, 7 y 8 tenían tasas inferiores a las óptimas en su adherencia médica, y no fue sino hasta la implementación del módulo de solución de problemas y pasos vitales que se apreció una mejoría de la misma. Estudios como el de Safren, González y Soroudi (2007), Safren et al. (2009) y Simoni et al. (2013) llevan a suponer que la mejoría en la adherencia ocurre debido a esos módulos específicos. Por ejemplo, Gross et al. (2013), trabajando con 218 participantes (38 excluidos, 91 en intervención basada en solución de problemas y 89 controles en tratamiento usual), documentaron que la solución de problemas es un componente medular para mejorar la adherencia terapéutica de los pacientes con VIH. Por su parte, el módulo de pasos vitales también ha mostrado su efectividad para aumentar los niveles de adherencia por sí sola (Psaros et al., 2014). Por tanto, se puede concluir que parte de los cambios en la adherencia se deben al efecto de dichos módulos.

Finalmente, los efectos de la intervención en los participantes 3,5 y 6 ratifican la premisa de una correlación inversa entre la adherencia terapéutica y la sintomatología depresiva (DiMatteo et al., 2000; Horberg et al., 2008; Maru et al., 2008; Orejudo et al., 2006; Piña et al., 2008; Starace et al., 2002). El participante 3 mejoró sus niveles de adherencia, pero no hasta alcanzar una que fuese prácticamente "perfecta" como en los otros casos, quizá por su nivel de sintomatología depresiva en el seguimiento. El participante 5 representa el caso contrario: una vez que se estabilizó su estado de ánimo (reflejado en su nivel de agrado semanal), aumentaron sus niveles de adherencia. Quizá el caso más claro de lo anterior es el del participante 6 , en el que es posible apreciar una disminución gradual de la sintomatología depresiva (y su nivel de agrado semanal) y un aumento de los niveles de adherencia. También se aprecian los efectos de los módulos de solución de problemas y de pasos vitales en los niveles de adherencia, los cuales alcanzaron el cien por ciento.

Al igual que todas las investigaciones de naturaleza clínica con las características del presente, este estudio tuvo algunas limitaciones. En primer lugar, los medios empleados para la captación de participantes pudo haber afectado su tipo y cantidad. Se puede suponer que solo los pacientes motivados al cambio y a ser adherentes decidieron acercarse al grupo de investigación para participar. En segundo lugar, los periodos en los que se llevó a cabo la intervención (entre ellos el sismo ocurrido en Ciudad de México en septiembre de 2017 y el periodos vacacional de diciembre del mismo año) pueden haber impedido que todos los participantes se expusieran exactamente a los mismos tiempos de intervención, e incluso aumentaran su deserción. En tercer lugar, el número de participantes reclutados para el estudio fue pequeño. En cuarto lugar, todos los participantes poseían pautas de medicación con tratamientos relativamente sencillos (una o dos tabletas al día tomadas a la misma hora), lo cual habría facilitado que los participantes presentaran mejores niveles de adherencia.

Por todo lo anterior, se sugiere realizar estudios futuros que permitan saber si el paso del tiempo afecta los resultados del módulo de $\mathrm{AC}$ al aumentar el tiempo entre consultas; examinen réplicas de los resultados de la presente investigación con un mayor número de participantes; posean una muestra con pautas médicas más complejas (un mayor número de tabletas por día, etc.); implementen la intervención comparando su instrumentación por parte de un personal capacitado y no especializado a fin de corroborar la efectividad de la misma sin el requisito de una alta especialidad 
profesional y a un menor costo; permitan hacer combinaciones en los módulos de la intervención para corroborar los efectos de cada uno en las variables de interés y pueda intentarse así un análisis de componentes, e incluyan finalmente una con- dición de comparación para corroborar la manera en que contribuye la intervención en las diversas duraciones de tratamiento a fin de aislar la posible contribución del paso del tiempo, en especial en aquellos pacientes con diagnóstico reciente.

\section{REFERENCIAS}

Balandrán, D.A., Gutiérrez, J.P. y Romero, M. (2013). Adherencia antirretroviral en México. Revista de Investigación Clínica, 65(5), 384-391.

Balfour, L., Kowal, J., Silverman, A., Tasca, G., Angel, J., Macpherson, P. y Cameron, D. (2006). A randomized controlled psycho-education intervention trial: improving psychological readiness for successful HIV medication adherence and reducing depression before initiating HAART. AIDS Care, 18(7), 830-838.

Bell, E., Marcus, D. y Goodlad, J. (2013). Are the parts as good as the whole? A meta-analysis of component treatment studies. Journal of Consulting and Clinical Psychology, 81(4), 722-736. doi: 10.1037/a0033004.

Berg, C., Michelson, S. y Safren, S. (2007). Behavioral aspects of HIV care: adherence, depression, substance use, and HIV-transmission behaviors. Infectious Disease Clinics, 21(1), 181-200.

Berg, C., Raminani, S., Greer, J., Harwood, M. y Safren, S. (2008). Participants' perspectives on cognitive-behavioral therapy for adherence and depression in HIV. Psychotherapy Research: Journal of the Society for Psychotherapy Research, 18(3), 271-280. doi: 10.1080/10503300701561537.

Bottonari, K., Roberts, J., Ciesla, J. y Hewitt, R. (2005). Life stress and adherence to antiretroviral therapy among HIV-positive individuals: a preliminary investigation. AIDS Patient Care \& Studies, 19(11), 719-727.

Calderón N., G. (1995). Un cuestionario para simplificar el diagnóstico del síndrome depresivo. Trabajo presentado en el homenaje rendido al doctor Carlos Alberto Seguín por la Asociación Psiquiátrica Peruana, Lima, 25 de agosto.

Castro, J., Gutiérrez, R. y Villena, J. (2007). Actualización para el médico no especialista en el tratamiento del VIH. Acta Médica Peruana, 24(3), 208-222.

Centro Nacional para la Prevención y el Control del VIH y el Sida (CENSIDA) (2017). Epidemiología / Registro Nacional de casos de VIH y sida. Disponible en https://www.gob.mx/cms/uploads/attachment/file/301432/RN_4to_trim_2017.pdf.

Costello, C.G. (1972). Depression: Loss of reinforcers of loss of reinforcer effectiveness? Behavior Therapy, 3(2), $240-247$.

Cruz J., B. (2015). Efecto de la depresión en la discapacidad en hombres con VIH en una clínica especializada. Tesis inédita. México: Universidad Nacional Autónoma de México.

Daughters, S.B., Braun, A., Sargeant, M., Reynolds, E., Hopko, D., Blanco, C. y Lejuez, C.W. (2008). Effectiveness of a brief behavioral treatment for innercity illicit drug users with elevated depressive symptoms: The life enhancement treatment for substance use (LETS Act!). Journal of Clinical Psychiatry, 69(1), 122-129.

Daughters, S.B., Magidson, J.F., Schuster, R. y Safren, S. (2010). Act healthy: A combined behavioral activation for depression and cognitive-behavioral HIV medication adherence treatment for substance users in residential treatment. Cognitive and Behavioral Practice, 17(3), 309-321.

DiMatteo, M., Lepper, H. y Croghan, T. (2000). Depression is a risk factor for noncompliance with medical treatment: Meta-analysis of the effects of anxiety and depression on patient adherence. Archives of Internal Medicine, 160(14), 21012107.

Dimidjian, S., Hollon, S., Dobson, K., Schmaling, K., Kohlenberg, R., Addis, M., Gallop, R., McGlinchey, J., Markley, D., Gollan, J., Atkins, D., Dunner, D. y Jacobson, N. (2006). Randomized trial of behavioral activation, cognitive therapy, and antidepressant medication in the acute treatment of adults with major depression. Journal of Consulting and Clinical Psychology, 74(4), 658-670. doi: 10.1037/0022-006X.74.4.658.

Ekers, D., Godfrey, C., Gilbody, S., Parrott, S., Richards, D.A., Hammond, D. y Hayes, A. (2011). Cost utility of behavioural activation delivered by the non-specialist. British Journal of Psychiatry, 199(5), 510-511. doi: 10.1192/bjp.bp.110.090266.

Fahrion, S.L. y Norris, P. A. (1990). Self-regulation of anxiety. Bulletin of the Menninger Clinic, 54, $217-231$.

Ferster, C.B. (1965). Classification of behavioral pathology. En L. Krasner y L.Ullman (Eds.): Research in behavior modification. New York: Holt, Rinehart \& Winston.

Ferster, C.B. (1973). A functional analysis of depression. American Psychologist, 28(10), 857-870.

Galindo. J. y Ortega, M. (2010). Prevalencia de depresión en pacientes con VIH/SIDA en el Hospital General de Zona Núm. 11 del Instituto Mexicano del Seguro Social (IMSS) “Lic. Ignacio Díaz Téllez”. Enfermedades Infecciosas y Microbiología, 30(4), 129-133. 
Galvan, F., Davis, E., Banks, D. y Bing, E. (2008). HIV stigma and social support among African Americans. AIDS Patient Care \& Studies, 22(5), 423-436. doi: 10.1089/apc.2007.0169.

Gardner, E., Burman, W., Steiner, J., Anderson, P. y Bangsberg, D. (2009). Antiretroviral medication adherence and the development of class-specific antiretroviral resistance. AIDS, 23(9), 1035-1046.

Garduño Z., C., Riveros R., A. y Sánchez-Sosa, J.J. (2010). Calidad de vida y cáncer de mama: Efectos de una intervención cognitivo-conductual. Revista Latinoamericana de Medicina Conductual, 1(1), 69-80.

Gawrysiak, M., Nicholas, C. y Hopko, D. (2009). Behavioral activation for moderately depressed university students: Randomized controlled trial. Journal of Counseling Psychology, 56(5), 468-475.

Golin, C.E., Liu, H., Hays, R., Miller, L., Beck, C.K., Ickovics, J., Kaplan, A.H. y Wenger, N. (2002). A prospective study of predictors of adherence to combination antiretroviral medication. Journal of General Internal Medicine, 17(10), 756-765. doi: 10.1046/j.1525-1497.2002.11214.x.

González D., A., Reséndiz, A. y Reyes L., I. (2015). Adaptation of the BDI-II in Mexico. Salud Mental, 38(4), 237-244. Disponible en http://doi.org/10.17711/SM.0185-3325.2015.033.

Gortner, E., Gollan, J., Dobson, K. y Jacobson, N. (1998). Cognitive-behavioral treatment for depression: relapse prevention. Journal of Consulting and Clinical Psychology, 66(2), 377-384.

Gross, R., Bellamy, S.L., Chapman, J., Han, X., O’Duor, J., Palmer, S.C., Houts, P.S., Coyne, J.C. y Strom, B.L. (2013). Managed problem solving for antiretroviral therapy adherence: a randomized trial. JAMA Internal Medicine, 173(4), 300-306. doi: http://doi.org/10.1001/jamainternmed.2013.2152.

Hammer, S., Saag, M., Schechter, M., Montaner, J., Schooley, R., Jacobsen, D. y Volberding, P. (2006). Treatment for adult HIV infection: recommendations of the International AIDS Society-USA panel. HIV Medicine, 14(3), 827-843.

Hopko, D.R., Funderburk, J.S., Shorey, R.C., McIndoo, C.C., Ryba, M.M., File, A.A., Benson, K. y Vitulano, M. (2013). Behavioral activation and problem-solving therapy for depressed breast cancer patients: preliminary support for decreased suicidal ideation. Behavior Modification, 37(6), 747-767. doi: 10.1177/0145445513501512.

Hopko, D.R., Lejuez, C.W., Ruggiero, K.J. y Eifert, G.H. (2003). Contemporary behavioral activation treatments for depression: procedures, principles, and progress. Clinical Psychology, 23(6), 699-717. doi: 10.1016/S0272-7358(03)00070-9.

Horberg, M., Silverberg, M., Hurley, L., Towner, W., Klein, D., Bersoff-Matcha, S., Weinberg, W.G., Antoniskis, D., Mogyoros, M., Dodge, W.T., Dobrinich, R., Quesenberry, C.P. y Kovach, D. (2008). Effects of depression and selective serotonin reuptake inhibitor use on adherence to highly active antiretroviral therapy and on clinical outcomes in HIV-infected patients. Journal of Acquired Immune Deficiency Syndrome, 47(3), 384-390.

Hughes, R., Sterne, J., Walsh, J., Bansi, L., Gilson, R., Orkin, C., Hill, T., Ainsworth, J., Anderson, J., Gompels, M., Dunn, D., Johnson, M.A., Phillips, A.N., Pillay, D., Leen, C., Easterbrook, P., Gazzard, B., Fisher, M. y Sabin, C. (2011). Long-term trends in CD4 cell counts and impact of viral failure in individuals starting antiretroviral therapy: UK Collaborative HIV Cohort (CHIC) study. HIV Medicine, 12(10), 583-593. doi: 10.1111/j.1468-1293.2011.00929.x.

Kanter, J., Diéguez, G., Rusch, L., Busch, A. y Santiago-Rivera, A. (2008). Behavioral activation for Latinos with depression. Clinical Case Studies, 7(5), 491-506.

Kazdin, A. (2011). Background and key measurements considerations. En A. Kazdin (Ed.): Single case research designs: Methods for clinical and applied settings (2nd ed.). New York: Oxford University Press.

Klimas, N., O’Brien, A. y Fletcher, M.A. (2008). Overview of HIV. Psychosomatic Medicine, 70(6), 523-530.

Lundervold, D., Talley, C. y Buermann, M. (2008). Effect of behavioral activation treatment on chronic fibromyalgia pain: replication and extension. International Journal of Behavioral Consultation and Therapy, 4(2), 146-157.

Magidson, J.F., Seitz-Brown, C.J., Safren, S.A. y Daughters, S.B. (2014). Implementing behavioral activation and life-steps for depression and HIV medication adherence in a community health center. Cognitive and Behavioral Practice, 21(4), 386403. doi: 10.1016/j.cbpra.2013.10.002.

Mao, L., Kippax, S., Newman, C., Andrews, G., Rogers, G., Saltman, D. y Kidd, M. (2008). Rates of depression among men attending high-HIV-caseload general practices in Australia. Mental Health in Family Medicine, 5(2), 79-83.

Maru, D., Bruce, R., Walton, M., Mezger, J., Springer, S., Shield, D. y Altice, F. (2008). Initiation, adherence, and retention in a randomized controlled trial of directly administered antiretroviral therapy. AIDS and Behavior, 12(2), $284-293$.

Newman, C., Limin, M., Kippax, S., Saltman, D. y Kidd, M. (2008). Management of HIV and depression in general practice: the Primary Health Care Project on HIV and Depression. HIV Australia, 7(4), 18-45.

Nezu, A. (1987). A problem-solving formulation of depression: A literature review and proposal of a pluralistic model. Clinical Psychology Review, 7(2), 121-144. doi: https://doi.org/10.1016/0272-7358(87)90030-4.

Nezu, A., Nezu, C., Saraydarian, L., Kalmar, K. y Ronan, G. (1986). Social problem solving as a moderating variable between negative life stress and depressive symptoms. Cognitive Therapy and Research, 10(5), 489-498.

Organización Mundial de la Salud (2013). Temas de salud: VIH/SIDA. Ginebra: OMS. Disponible en http://www.who.int/topics/ hiv_aids/es/. 
Organización Mundial de la Salud (2017). VIH/sida. Ginebra: OMS. Disponible en http://www.who.int/mediacentre/factsheets/ fs $360 / \mathrm{es} /$.

Orejudo, S., Ladero, L., Carrobles, J.A. y Malo, C.C. (2006). Adherencia al tratamiento antirretroviral y ansiedad, depresión y estrés en sujetos en un programa de mantenimiento con metadona. Ansiedad y Estrés, 12(1), 19-29.

Peñarrieta M., I., Kendall, T., Martínez, N., Rivera A., M., González, N., Flores, F. y Ángel E., D. (2009). Adherencia al tratamiento antirretroviral en personas con VIH en Tamaulipas, México. Revista Peruana de Medicina Experimental y Salud Pública, 26(3), 333-337.

Pinto, M., Cruz, M., Tavares, C., Silveira, T., Sica, A. y Beltrami, L. (2012). Depressive symptoms in HIV-infected patients treated with highly active antiretroviral therapy. Revista Brasileira de Psiquiatria, 34(2), 162-167.

Piña, J.A. y Sánchez-Sosa, J.J. (2007). Modelo psicológico para la investigación de los comportamientos de adhesión en personas con VIH. Universitas Psychologica, 6(2), 399-407.

Piña, J.A., Corrales A., E., Mungaray, K. y Valencia M., A. (2006). Instrumento para medir variables psicológicas y comportamientos de adhesión al tratamiento en personas seropositivas frente al VIH (VPAD-24). Revista Panamericana Salud Pública, 19(4), 217-228.

Piña, J.A., Dávila T., M, Sánchez-Sosa, J.J., Togawa, C. y Cázares R., O. (2008). Association between stress and depression levels and treatment adherence among HIV-positive individuals in Hermosillo, México. Revista Panamericana de Salud Pública, 23(6), 377-383.

Piña, J. A., Valencia, M. A., Mungaray, K. y Corrales, A. E. (2006). Validación de una escala breve que mide situaciones vinculadas con estrés en personas con VIH. Terapia Psicológica, 24(1), 15-21.

Psaros, C., Haberer, J.E., Katabira, E., Ronald, A., Tumwesigye, E., Campbell, J.D. y Safren, S.A. (2014). An intervention to support HIV pre-exposure prophylaxis (PrEP) adherence in HIV serodiscordant couples in Uganda. Journal of Acquired Immune Deficiency Syndromes, 66(5), 522-529. doi: http://doi.org/10.1097/QAI.0000000000000212.

Rodkjaer, L., Laursen, T., Balle, N. y Sodemann, M. (2010). Depression in patients with HIV is under-diagnosed: a cross-sectional study in Denmark. HIV Medicine, 11(1), 46-53. doi: 10.1111/j.1468-1293.2009.00741.x.

Safren, S., González, J. y Soroudi, N. (2007). Coping with chronic illness: Cognitive behavioral therapy for adherence and depression in individuals with chronic illness. Therapist Guide. New York: Oxford University Press.

Safren, S., O'Cleirigh, M., Bullis, J., Otto, M., Stein, M. y Pollack, M. (2012). Cognitive behavioral therapy for adherence and depression (CBT-AD) in HIV-infected injection drug users: A randomized controlled trial. Journal of Consulting and Clinical Psychology, 80(3), 404-415. doi: 10.1037/a0028208.

Safren, S., O'Cleirigh, M., Reilly, L., Tan, J., Raminani, S., Otto, M. y Mayer, K. (2009). A randomized controlled trial of cognitive behavioral therapy for adherence and depression (CBT-AD) in HIV-infected individuals. Health Psychology, 28(1), 1-10.

Safren, S., Otto, M. y Worth, J. (1999). Life-steps: Applying cognitive behavioral therapy to HIV medication adherence. Cognitive and Behavioral Practice, 6(4), 332-341. doi: 10.1016/S1077-7229(99)80052-2.

Safren, S., Otto, M., Worth, J., Salomon, E., Johnson, W., Mayer, K. y Boswell, S. (2001). Two strategies to increase adherence to HIV antiretroviral medication: Life-steps and medication monitoring. Behaviour Research and Therapy, 39(10), 1151-1162.

Sánchez-Sosa, J.J., Cázares, O., Piña, J.A. y Dávila, M. (2009). Un modelo psicológico en los comportamientos de adhesión terapéutica en personas con VIH. Salud Mental, 32(4), 389-397.

Santiago-Rivera, A., Kanter, J., Benson, G., Derose, T., Illes, R. y Reyes, W. (2008). Behavioral activation as an alternative treatment approach for latinos with depression. Psychotherapy Theory, Research, Practice \& Training, 45(2), 173-185.

Shuter, J. (2008). Forgiveness of non-adherence to HIV-1 antiretroviral therapy. Journal of Antimicrobial Chemotherapy, 61(4), 769-773.

Sidman, M. 1960. Tactics of scientific research; Evaluating experimental data in psychology. New York: Basic Books.

Simoni, J., Wiebe, J., Sauceda, J., Huh, D., Sanchez, G., Longoria, V., Andres B., C. y Safren, S. (2013). A preliminary RCT of CBT-AD for adherence and depression among HIV-positive Latinos on the U.S.-Mexico border: the Nuevo Día study. AIDS Behavior, 17(8), 2816-2819. doi: 10.1007/s10461-013-0538-5.

Soto, J. y Cruz, J. (2014). Depresión en pacientes con diagnóstico de VIH/SIDA. CEcorfan. Disponible en http://www.ecorfan.org/ handbooks/Educacion\%20T-IV/ARTICULO\%201.pdf.

Secretaría de Salud y Centro Nacional para la Prevención y el Control del VIH y el Sida (2008). Panorama epidemiológico del VIH/SIDA e ITS en México. Disponible en http://www.inegi.org.mx/prod_serv/contenidos/espanol/bvinegi/productos/integracion/sociodemografico/mujeresyhombres/2008/myh2008feb28_2.pdf.

Starace, F., Ammassari, A., Trotta, M., Murri, R., De Longis, P., Izzo, C., Scalzini, A., d'Arminio M., A., Wu, A.W. y Antinori, A. (2002). Depression is a risk factor for suboptimal adherence to highly active antiretroviral therapy. Journal of Acquired Immune Deficiency Syndromes, 31(5), 136-139.

Tate, R., Perdices, M., Rosenkoetter, U., Wakim, D., Godbee, K., Togher, L. y McDonald, S., (2013). Revision of a method quality rating scale for single-case experimental designs and $\mathrm{n}$-of-1 trials: The 15-item Risk of Bias in N-of-1 Trials (RoBiNT) Scale. Neuropsychological Rehabilitation, 23(5), 619-638. doi: 10.1080/09602011.2013.824383. 
Thomason, B., Bachanas, P. y Campos, P. (1996). Cognitive behavioral interventions with persons affected by HIV/AIDS. Cognitive and Behavioral Practice, 3(2), 417-442. doi: 10.1016/S1077-7229(96)80026-5.

U.S. Department of Health \& Human Services (2017). Limitations to treatment safety and efficacy adherence to the continuum of care. Disponible en https://aidsinfo.nih.gov/guidelines/html/1/adult-and-adolescent-arv/30/adherence.

Uthman, O.A., Magidson, J.F., Safren, S.A. y Nachega, J.B. (2014). Depression and adherence to antiretroviral therapy in low-, middle- and high-income countries: a systematic review and meta-analysis. Current HIV/AIDS Reports, 11(3), 291-307.

Valente, S. (2003). Depression and HIV disease. The Journal of the Association of Nurses in AIDS Care (JANAC), 14(2), 41-51.

Vargas, C. y Boza, R. (2012). Condición inmunológica de los pacientes portadores de VIH/sida en el momento de su diagnóstico en el Hospital San Juan de Dios. Acta Médica Costarricense, 54(3) 159-164.

World Health Organization (2016). Consolidated guidelines on the use of antiretroviral drugs for treating and preventing HIV infection. Recommendations for a public health approach (2nd ed.). Geneve: Author. Disponible en http://apps.who.int/ iris/bitstream/handle/10665/208825/9789241549684_eng.pdf;jsessionid=59FF20669FF289231F4E543D83145155?sequence $=1$. 